\title{
Aggressive B-cell lymphoma: chasing the target
}

\author{
Jan Walewski
}

Lymphoid Malignancy, Maria Sklodowska-Curie Institute Oncology Center, Warszawa, Poland

\section{Correspondence to} Professor Jan Walewski, Lymphoid Malignancy, Maria Sklodowska-Curie Institute of Oncology Warsaw, Warszawa 02-034, Poland; jan.walewski@coi.pl

Accepted 2 October 2019 Published Online First 30 October 2019

\section{SLinked}

http://dx.doi.org/10. 1136/jim-2019-001266

\section{Check for updates}

(C) American Federation for Medical Research 2020. No commercial re-use. See rights and permissions. Published by BMJ.

To cite: Walewski J.

$J$ Investig Med

2020;68:331-334.

\section{ABSTRACT}

One of the first achievements of molecular biology

in lymphoma science was a discovery of cell of origin (COO) classification around 20 years ago with defining activated $B$-cell like $(A B C)$ and germinal center B-cell like subtypes of diffuse large B-cell lymphoma (DLBCL) with the use of gene expression profiling. These categories were considered important as seemed to present different biology, response to treatment, and prognosis. Immunochemotherapy R-CHOP21 has been a standard of care for 2 decades, and it results in long-term disease-free survival or cure of $60 \%$ of patients with DLBCL but efficacy in an individual patient depends on age and other International Prognostic Index clinical risk factors and is within a range of $30 \%$ to more than $90 \%$. Clinical attempts to enhance activity of immunochemotherapy in high-risk DLBCL like $A B C$ or others included adding targeted agents to the R-CHOP backbone: bortezomib, lenalidomide, ibrutinib. Unfortunately, randomized clinical trials did not confirm the expected benefit. Recently, advanced molecular techniques were used to classify B-cell lymphomas beyond $\mathrm{COO}$ or MYC alterations and correlated with clinical outcome as illustrated by 2 recently published influential studies from the National Institutes of Health and from Dana Farber Cancer Center, USA. Advanced molecular pathogenesis descriptions of DLBCL provide a framework for actionable classifications that should be used for designing future clinical trials and hopefully bring success to treatment of high-risk aggressive lymphoma.

Current classification of lymphoid neoplasms updated in $2016^{1}$ identifies 22 clinical and pathological disease entities of $\mathrm{B}$-cell origin that are clinically aggressive. They constitute around one-quarter of the new cases of the lymphoid neoplasms, ${ }^{2}$ including chronic lymphocytic leukemia, Hodgkin's lymphoma and plasma cell myeloma. The most frequent is diffuse large B-cell lymphoma-not otherwise specified (DLBCL-NOS), and treatment recommendations for aggressive lymphoma are based on data derived from this entity and extrapolated to other disease subtypes except for a few that were specifically investigated including primary central nervous system lymphoma, primary mediastinal large B-cell lymphoma, and Burkitt lymphoma. New entities of particular interest are defined based on accumulated genetic and clinical data justifying their distinction: high-grade B-cell lymphoma (HGBL) with MYC and $B C L 2$ and/or BCL6 rearrangement, ${ }^{34}$ and HGBL-NOS, as well as DLBCL-NOS subtypes based on cell of origin (COO): germinal center B-cell like (GCB) and activated B-cell like (ABC). Growing clinical evidence suggests a need for individualized approach to these entities. $^{134}$

Immunochemotherapy R-CHOP21 has been a standard of care for 2 decades, and it results in long-term disease-free survival or cure of $60 \%$ of patients with DLBCL but efficacy in an individual patient depends on age and other International Prognostic Index (IPI) clinical risk factors and is within a range of $30 \%$ to more than 90\%. National Comprehensive Cancer Network and British Columbia Cancer Agency recently validated the prognostic value of the IPI in patients with DLBCL treated with R-CHOP in 2000-2010 period. $^{5}$ The prognostic value of all 5 factors including age, performance score, disease stage, elevation of lactate dehydrogenase (LDH), and extranodal involvement was confirmed and age and LDH level were subdivided into ranges to account for a continuous negative influence of these variables on survival. In addition, confirmed negative prognostic influence of particular extranodal sites (E) including bone marrow, central nervous system (CNS), gastrointestinal tract and liver, and lung but not the $\mathrm{E}$ number itself.

One of the first achievements of molecular biology in lymphoma science was a discovery of COO classification around 20 years ago with defining $A B C$ and GCB subtypes with the use of gene expression profiling (GEP). These categories were considered important as seemed to present different biology, response to treatment, and prognosis. Given that GEP is not generally available for routine diagnostics, it is acceptable to use one of several immunohistochemical (IHC) algorithms, mostly Hans algorithm, for discriminating GCB and non-GCB subtypes: $\mathrm{GCB}=\mathrm{CD} 10+$ or $\mathrm{CD} 10-$ and BCL6+; non$\mathrm{GCB}=\mathrm{CD} 10$ and $\mathrm{BCL} 6-$ or BCL6+ and MUM-1+. Accuracy of the IHC methods is however only around $80 \%$.

Some retrospective data indicated worse outcome of patients with $\mathrm{ABC} /$ non-GCB DLBCL treated with R-CHOP compared with patients with GCB lymphoma.

ABC-DLBCL subtype is believed to originate from the B-cell that underwent germinal center reaction and is committed to plasmablast differentiation. ${ }^{1} \mathrm{ABC}$ lymphomas demonstrate 
increased activity of NF- $\kappa \mathrm{B}$, genetic alterations of NF- $\mathrm{BB}$ modifiers and $\mathrm{B}$-cell receptor (BCR) signaling pathway elements, as well as disturbed terminal differentiation.

GCB lymphomas likely originate from the light zone of the germinal center, may have alterations of the chromatinmodifying enzymes, PI3K signaling disturbances, and BCL2 structural variants.

Data on predominant molecular alterations and deteriorated response to treatment of $\mathrm{ABC}$ compared with $\mathrm{GCB}$ lymphoma were the major indications for prospective clinical trials of agents targeted to these alterations. ${ }^{6}$ There were a number of reasons to believe that adding proteasome inhibitor bortezomib, which is able to inhibit nuclear translocation and transcriptional activity of NF- $\mathrm{KB}$, to R-CHOP backbone, will likely improve outcome in $\mathrm{ABC}$ lymphoma subtype.

A randomized phase II study PYRAMID ${ }^{7}$ involving 206 patients with a diagnosis of non-GCB DLBCL established by the use of Hans algorithm compared R-CHOP with VR-CHOP (bortezomib added at $1.3 \mathrm{mg} / \mathrm{m}^{2}$ intravenous days 1 and 4) failed to show statistically significant improvement of progression-free survival (PFS). In addition, the outcome in a reference arm R-CHOP was markedly better than assumed in this patient population (2-year PFS 77.6\% after R-CHOP and $82.0 \%$ after VR-CHOP).

A randomized phase III REMoDL-B study ${ }^{8}$ involved 1128 previously untreated patients with DLBCL in need of systemic therapy. All patients received 1 initial cycle of $\mathrm{R}-\mathrm{CHOP}$ and diagnostic tissue sample was submitted to central pathology review with whole genome expression analysis for determining of the $\mathrm{COO}$ and classifying the case as either ABC, GCB, or unclassifiable DLBCL.

Patients were randomized 1:1 to R-CHOP or RB-CHOP arm (added bortezomib at $1.3 \mathrm{mg} / \mathrm{m}^{2}$ intravenous or $1.6 \mathrm{mg} / \mathrm{m}^{2}$ subcutaneous days 1 and 8 of cycles 2-6) and stratified by the IPI risk factors and COO subtype. If the quality or quantity of RNA in diagnostic sample was suboptimal (14.7\% of cases), the patient was given standard treatment with R-CHOP. It was the first major study in patients with DLBCL using real-time molecular characterization for prospective screening, stratifying, randomization, and final analysis of data in biologically defined patient subgroups. However, adding bortezomib did not improve PFS. Contrary to expectations, bortezomib proved not to be an effective inhibitor of NF- $\mathrm{KB}$ pathway in ABC-DLBCL. Even in cases with identified somatic mutations of genes related to NF- $\mathrm{KB}$ activation like CARD11, CD79A/B, MYD88, TNFAIP3, TNFRSF11A, PFS was identical in both study arms. On the other hand, explorative subgroup analysis suggests that proteasome inhibitor may positively influence the outcome in other high-risk subsets of DLBCL like double hit and double expressor what needs to be addressed by appropriate clinical study. Design of the REMoDL-B study is a useful model for future investigations of new targeted agents for patients with DLBCL. Utility of $\mathrm{R}-\mathrm{CHOP}$ regimen as a backbone for new protocols was also confirmed by this study.

Another rational approach to improving activity of immunochemotherapy was developing of more active anti-CD20 antibody. Obinutuzumab is a type II antibody with a glycosyl moiety engineered by means of fructose deletion that demonstrates increased ability to induce antibody-dependent cellular cytotoxicity and lysosome-dependent cell death with attenuated activation of complement-dependent cytotoxicity.

A recently published randomized study $\mathrm{GOYA}^{9}$ in patients with advanced DLBCL with 2 or more IPI risk factors and/or presence of bulky disease directly comparing PFS of patients treated with obinutuzumab or rituximab both combined with CHOP showed no difference: 3-year PFS of $70 \%$ and $67 \%$ for G-CHOP and R-CHOP, respectively.

A concept of a prolonged 96 hours' continuous infusion of cytotoxic agents with a dose adjustment to the degree of hematologic toxicity had been explored for decades at the National Cancer Institute, USA, with a variable success and recently considered potentially active in high-risk aggressive lymphoma did not prove significantly superior to R-CHOP in recently published randomized study ${ }^{10}$ with 2-year PFS of $78.9 \%$ and $75.5 \%$ for DA-EPOCH-R and R-CHOP, respectively, and with markedly increased toxicity of the infusional regimen including infection, neutropenic fever, mucositis, and neuropathy.

Another promise was brought with Bruton's tyrosine kinase inhibitor ibrutinib suppressing BCR signaling that was shown preferentially active against ABC-DLBCL in monotherapy in a phase I/II study ${ }^{11}$ in recurrent/refractory disease (overall response rate 37\%) as well as safe in a combination with R-CHOP in a phase I study ${ }^{12}$ for previously untreated patients. However, a double-blind, randomized study PHOENIX ${ }^{13}$ designed to compare eventfree survival (EFS) in patients with DLBCL of non-GCB subtype centrally determined by Hans-based immunohistochemistry and IPI score of 1 or more treated with R-CHOP plus ibrutinib or R-CHOP plus placebo did not show expected improvement with ibrutinib. Interestingly, statistically significant interaction between treatment and age was found. In patients younger than 60 , ibrutinib significantly improved EFS, PFS, and overall survival (OS) (HR 0.579, 0.556 , and 0.330 , respectively) and increased incidence of serious adverse events $(35.7 \%$ vs $28.6 \%)$. In patients aged 60 years or older, adding ibrutinib to R-CHOP decreased EFS, PFS, and OS, increased incidence of serious adverse events $(63.4 \%$ vs $38.2 \%)$, and decreased treatment compliance. The authors conclude that the study did not meet its primary endpoint in the intent-to-treat (ie, non-GCB) or $\mathrm{ABC}$ patients (as determined by the retrospective GEP analysis) but the interaction of age with treatment needs further investigation.

Immunomodulatory agent lenalidomide has been tested in several B-cell lymphoma types based on mechanistic rationale including reduction of interferon regulating factor-4 needed for plasmablastic differentiation and cell survival as well as derepression of interleukin- 2 synthesis. In addition, some phase II data suggested that lenalidomide may reverse the negative prognostic impact of the $\mathrm{ABC}$ phenotype.

A randomized phase III study ROBUST ${ }^{14}$ included untreated patients with ABC DLBCL (CD20+) subtype prospectively determined with the use of NanoString Lymphoma Subtyping test based on gene expression analysis technique Lymph2Cx (Scott, Blood 2014). Eligible were patients with IPI score of 2 or more and were randomized to the standard treatment R-CHOP21 plus placebo or lenalidomide $15 \mathrm{mg}$ oral days 1-14 to 6 cycles and 2 additional doses of rituximab according to local practice. PFS, the primary endpoint, was similar in both arms (HR 0.85; 95\% CI 0.63 to $1.14 ; \mathrm{p}=0.29$ ), 
although R2CHOP showed tendency to better outcome in patients with more advanced clinical stage and IPI score $\geq 3$. ORR was $91 \%$ in both arms, complete response (CR) 69\% and $65 \%$ in $\mathrm{R} 2 \mathrm{CHOP}$ and R-CHOP arms, respectively. All 6 cycles of treatment completed $74 \%$ and $84 \%$ of patients in both arms, respectively. The most frequent cause of treatment discontinuation was neutropenia.

A similar randomized but phase II study with lenalidomide ECOG-ACRIN1412 15 involved patients with DLBCL regardless of $\mathrm{COO} \mathrm{ABC}$ or GCB with similar to ROBUST study clinical risk factors (IPI $\geq 2$, Eastern Cooperative Oncology Group Performance Status $\leq 2$ ). COO classification was performed with the use of the same method of gene expression analysis NanoString Lymph2Cx with the aim of evaluating patient outcome in ABC DLBCL subtype. Patients were randomized to standard R-CHOP21 treatment or lenalidomide added to R-CHOP2 1 at $25 \mathrm{mg}$ oral days $1-10$ to 6 cycles. The primary endpoint was PFS. In this study, adding lenalidomide to R-CHOP was associated with 33\% reduction of PFS risk and was statistically significant (HR $0.67 ; 95 \%$ CI 0.44 to 1.03 ; $\mathrm{p}=0.03$ (one sided)). Based on COO, PFS HR for R2CHOP was: 0.68 for $\mathrm{ABC}, \mathrm{p}=0.15,0.86$ for $\mathrm{GCB}, 0.83$ for unclassified, and 0.61 for unknown cases. Objective response and CR rate was similar in R-CHOP and R2CHOP arms of 92\% and $67 \%$, and $97 \%$ and $72 \%$, respectively (p NS). The 2 -year OS was $87 \%$ and $80 \%$, respectively. Toxicity was as expected for R-CHOP with significantly different rates of grade 3 or more adverse events for diarrhea ( $6 \%$ vs $0.6 \%, \mathrm{p}=0.005$ ), febrile neutropenia ( $25 \%$ vs $12 \%, \mathrm{p}=0.003)$, and thrombocytopenia ( $36 \%$ vs $12 \%, \mathrm{p}<0.0001$ ) in R2CHOP versus R-CHOP arm, respectively. In conclusion, contrary to the ROBUST phase III study, the addition of lenalidomide to R-CHOP in this phase II study improved PFS in newly diagnosed DLBCL.

It is intriguing that these 2 similar studies resulted in a different conclusion. However, there were differences between the studies: phase II versus phase III, target patient cohort non-GCB versus DLBCL unspecified, lenalidomide dosing $25 \mathrm{mg} / 10$ days vs $15 \mathrm{mg} / 14$ days. Taken together, it should be stated that R2CHOP regimen is not yet ready as alternative for patients with DLBCL. In addition, COO classification of $A B C$ and GCB subtypes based on GEP techniques may not be precise enough to guide treatment choice, and thus explain why the clinical trials with agents targeting COO subtypes failed.

Among new aggressive lymphoma cases, around onethird show alterations of $c$-MYC oncogene including rearrangements, gain of copy number or increase of MYC protein expression. MYC rearrangement can be found in $12 \%$ of patients, and in around $8 \%$ of cases it is associated with BCL2 and/or BCL6 rearrangements. These double-hit and triple-hit lymphomas are now classified as HGBL with $M Y C$ and BCL2 and/or BCL6 rearrangements. It is generally believed that these lymphomas are associated with poor prognosis if the patients are treated with $\mathrm{R}-\mathrm{CHOP}$, present more frequently with poor risk factors, and are at higher risk of CNS involvement. Reports from single centers suggested better outcome if the patients received intensive induction treatment. A role of high-dose chemotherapy and autologous hematopoietic cell transplantation as a consolidation of remission is not clear. Cases of protein MYC and BCL2 overexpression referred to as 'double expressor DLBC' are believed to be associated with poor prognosis as well. ${ }^{6}$
Recently, advanced molecular techniques were used to classify B-cell lymphomas beyond COO or MYC alterations and correlated with clinical outcome as illustrated by 2 recently published influential studies. A group from the National Institutes of Health, USA, ${ }^{16}$ reported results of exome and transcriptome sequencing, targeted amplicon resequencing, and array-based DNA copy number analysis of 372 genes from the fresh biopsy samples of 574 patients with DLBCL to identify genes with recurrent aberrations. ${ }^{16}$ The authors developed and implemented an algorithm that was able to assign $47 \%$ of cases to 4 prominent genetic subtypes in DLBCL based on the co-occurrence of genetic alterations. The subtypes were termed:

- MCD (co-occurrence of MYD88 $8^{\mathrm{L} 265 \mathrm{P}}$ and $C D 79 B$ mutations).

- BN2 (BCL6 fusions and NOTCH2 mutations).

- N1 (NOTCH1 mutations)/.

- EZB (EZH2 mutations and BCL2 translocations).

These subtypes differed phenotypically by differences in gene expression signatures and response to treatment with favorable survival in the BN2 and EZB subtypes and inferior outcome in $\mathrm{MCD}$ and N1 subtypes. Genetic pathway analysis suggested that MCD and BN2 subtypes depended on chronic active BCR signaling that is theoretically susceptible to therapeutic inhibition. Analyzing cases by $\mathrm{COO}$ according to GEP showed that $23.1 \%$ and $13.6 \%$ of $\mathrm{ABC}$ cases were classified as MCD and BN2, respectively, and $37.2 \%$ and $11.6 \%$ of GCB cases as EZB and BN2, respectively. However, the majority of both $\mathrm{ABC}$ and GCB cases were unclassified. Survival analysis of 119 patients treated with R-CHOP or similar regimen in genetic subtypes showed 5 -year OS rates of $26 \%, 36 \%, 65 \%$, and $68 \%$ for MCD, N1, BN2, and EZB subgroups, respectively.

A group from Dana Farber Cancer Institute, USA, analyzed 304 biopsy samples from untreated patients with DLBCL who subsequently received R-CHOP regimen. A study involved a comprehensive genetic analysis, identifying low-frequency alterations, capturing recurrent mutations, somatic copy number alterations, and structural variants, and defining coordinate signatures in patients with available outcome data. The genetic drivers were integrated using consensus clustering, and 5 robust DLBCL subsets called 'clusters' were identified: C1-C5. ${ }^{17}$ Around 96\% of cases were able to be classified into 5 clusters (C) that were defined based on predominant alterations:

C1-BCL6 structural variants, NOTCH2 mutations, mutations of NF- $\mathrm{kB}$ pathway elements: BCL10, TNFAIP3(A20) and FAS, alterations responsible for immune escape including inactivating mutations of $B 2 M, C D 70, F A S$ and structural variants of PD-L1, PD-L2.

C2-biallelic inactivating mutations of TP53 and $17 p$ copy loss, copy loss of 9p21.13/CDKN2A and 13q14.2/ $R B 1$, which perturb chromosomal stability and cell cycle.

C3-BCL2 mutations with concordant structural variants that juxtapose $B C L 2$ to the $I g H$ enhancer, frequent mutations in chromatin modifiers, KMT2D, CREBBP, EZH2, as well as PTEN inactivating alterations.

C4-mutations in histone genes, multiple immune evasion molecules CD83, CD58, CD70, BCR/Pi3K signaling intermediates (RHOA, GNA13, SGK1), NF-KB modifiers (CARD11, NFKBIE, NFKBIA) and RAS/JAK/STAT pathway members (BRAF, STAT3). 


\begin{tabular}{ll}
\hline $\begin{array}{l}\text { Table } 1 \\
\text { Relation of commercially available therapeutic agents } \\
\text { and new molecular subtypes }\end{array}$ & \\
\hline DLBCL molecular subtype & Potential therapeutic agent \\
\hline MCD/C5 & Ibrutinib, acalabrutinib, venetoclax \\
\hline BN2/C1 & $\begin{array}{l}\text { Ibrutinib, bortezomib, carfilzomib } \\
\text { Venetoclax, idelalisib, copanlisib, } \\
\text { duvelisib }\end{array}$ \\
EZB/C3 & $\begin{array}{l}\text { Idelalisib, copanlisib, duvelisib, } \\
\text { bortezomib, carfilzomib, ruxolitinib }\end{array}$ \\
\hline
\end{tabular}

DLBCL, diffuse large B-cell lymphoma.

C5-18q gain increasing expression of BCL2 and MALT1, frequent mutations in CD79B and MYD $88^{L 265 P}$, gains of 3q, 19q13.42 and inactivation of PRMD1, 18p copy gains. These alterations along with additional mutations in ETV6, PIM1, GRHPRTBL1XR1, and BTG1 are similar to those described in primary CNS and testicular lymphoma, thus implicating that the $\mathrm{C} 5$ genetic signature is associated with extranodal tropism.

When correlated with $\mathrm{COO}, \mathrm{C} 1$ and $\mathrm{C} 5$ signatures correlated with $\mathrm{ABC}$, and $\mathrm{C} 3$ and $\mathrm{C} 4$ with GCB. C5 signature corresponds to the unfavorable part of $\mathrm{ABC}$, and $\mathrm{C} 3$ signature to the unfavorable part of GCB.

Comparing these 2 genetic classifications suggests similarities between molecular subtypes, in particular MCD with $\mathrm{C} 5$, BN2 with $\mathrm{C} 1$, and EZB with C3. Advanced molecular pathogenesis descriptions of DLBCL provide a framework for actionable classifications that should be used for designing future clinical trials and hopefully bring success to treatment of high-risk aggressive lymphoma.

The potential match of potential therapeutic targeted agents and new molecular subtypes is summarised in table 1.

Contributors JW conceived, wrote, and edited the manuscript.

Funding The authors have not declared a specific grant for this research from any funding agency in the public, commercial or not-for-profit sectors.

Competing interests JW: Advisory role: Roche, Celgene, Takeda, JanssenCilag, Servier, Amgen, BMS, AbbVie, Novartis, Gilead. Research funding: Roche, GSK/Novartis, Takeda, Janssen-Cilag. Lecture honoraria: Roche, Celgene, Takeda, Janssen-Cilag, Servier, Amgen, AbbVie, Gilead. Conference travel support: Roche.

Patient consent for publication Not required.

Provenance and peer review Commissioned; externally peer reviewed.

ORCID iD

Jan Walewski http://orcid.org/0000-0003-4247-2674

\section{REFERENCES}

1. Swerdlow SH, Campo E, Pileri SA, et al. The 2016 revision of the World Health Organization classification of lymphoid neoplasms. Blood 2016;127:2375-90.

2. Wojciechowska U, Czaderny K, Ciuba A, et al. Nowotwory złośliwe w Polsce w 2016 roku. Krajowy Rejestr Nowotworów, Zakład Epidemiologii i Prewencji Nowotworów, Centrum Onkologii - Instytut im. Marii Skłodowskiej-Curie, Narodowy Program Zwalczania Chorób. Warszawa: Nowotworowych, Ministerstwo Zdrowia, 2018.

3. Sha C, Barrans S, Cucco F, et al. Molecular high-grade B-cell lymphoma: defining a poor-risk group that requires different approaches to therapy. J Clin Oncol 2019;37:202-12.

4. Ennishi D, Jiang A, Boyle M, et al. Double-hit gene expression signature defines a distinct subgroup of germinal center B-cell-like diffuse large B-cell lymphoma. JCO 2019:37:190-201.

5. Zhou Z, Sehn LH, Rademaker AW, et al. An enhanced International prognostic index (NCCN-IPI) for patients with diffuse large B-cell lymphoma treated in the rituximab era. Blood 2014;123:837-42.

6. Kahl BS, Landsburg DJ. Exploiting the molecular heterogeneity of aggressive B-cell lymphomas to develop effective new therapies., 2019. Available: https:// dailynews.ascopubs.org/do/10.1200/ADN.19.190191/full/?cid=DM2180\&bid= 16934439

7. Leonard JP, Kolibaba KS, Reeves JA, et al. Randomized phase II study of $\mathrm{R}$-CHOP with or without bortezomib in previously untreated patients with non-germinal center B-cell-like diffuse large B-cell lymphoma. J Clin Oncol 2017:35:3538-46.

8. Davies A, Cummin TE, Barrans S, et al. Gene-expression profiling of bortezomib added to standard chemoimmunotherapy for diffuse large B-cell lymphoma (REMoDL-B): an open-label, randomised, phase 3 trial. Lancet Oncol 2019:20:649-62.

9. Vitolo $U$, Trněný $M$, Belada $D$, et al. Obinutuzumab or rituximab plus cyclophosphamide, doxorubicin, vincristine, and prednisone in previously untreated diffuse large B-cell lymphoma. J Clin Oncol 2017;35:3529-37.

10. Bartlett NL, Wilson WH, Jung S-H, et al. Dose-Adjusted EPOCH-R compared with R-CHOP as frontline therapy for diffuse large B-cell Lympho-ma: clinical outcomes of the phase III intergroup trial Alliance/CALGB 50303. J Clin Oncol 2019;37:1790-9.

11. Wilson WH, Young RM, Schmitz R, et al. Targeting B cell receptor signaling with ibrutinib in diffuse large B cell lymphoma. Nat Med 2015;21:922-6.

12. Younes $A$, Thieblemont $C$, Morschhauser $F$, et al. Combination of ibrutinib with rituximab, cyclophosphamide, doxorubicin, vincristine, and prednisone (R-CHOP) for treatment-naive patients with CD20-positive B-cell nonHodgkin lymphoma: a non-randomised, phase 1B study. Lancet Oncol 2014;15:1019-26.

13. Younes A, Sehn LH, Johnson P, et al. Randomized phase III trial of ibrutinib and rituximab plus cyclophosphamide, doxorubicin, vincristine, and prednisone in Non-Germinal center B-cell diffuse large B-cell lymphoma. J Clin Oncol 2019;37:1285-95.

14. Vitolo U, Witzig TE, Gascoyne RD, et al. ROBUST: First report of phase III randomized study of lenalidomide/R-CHOP ( ${ }^{2}$-CHOP) vs placebo/R-CHOP in previously untreated $\mathrm{ABC}$-type diffuse large B-cell lymphoma. Hematol Oncol 2019;37:36-7.

15. Nowakowski GS, Hong F, Scott DW, et al. Addition of lenalidomide to RCHOP (R2CHOP) improves outcomes in newly diagnosed diffuse large B-cell lymphoma (DLBCL): first report of ECOG-ACRIN1412 a randomized phase 2 us intergroup study of R2CHOP vs R-CHOP. Hematol Oncol 2019;37:37-8.

16. Schmitz R, Wright GW, Huang DW, et al. Genetics and pathogenesis of diffuse large B-cell lymphoma. N Engl J Med 2018;378:1396-407.

17. Chapuy B, Stewart C, Dunford AJ, et al. Molecular subtypes of diffuse large $B$ cell lymphoma are associated with distinct pathogenic mechanisms and outcomes. Nat Med 2018;24:679-90. 